\title{
Two-step blind phase-shifting interferometry based on the extreme value of interference and least-squares iterative algorithm
}

\author{
Xiaoling $\mathrm{XU}^{1}$, Ming $\mathrm{XIE}^{2}$,Ying $\mathrm{JI}^{2}$, YaWei Wang ${ }^{2}$ \\ ${ }^{1}$ Institute of Mold Technology, Changzhou Vocational Institute of Mechatronic Technology, \\ Changzhou 213164, Jiangsu, China \\ ${ }^{2}$ Faculty of Science, Jiangsu University, Zhenjiang 212013, China \\ ${ }^{*}$ Corresponding author: xxqyzzd@sina.com
}

\begin{abstract}
To improve the measuring accuracy in two-step phase-shifting interferometry (PSI), a new approach combining the extreme value of interference (EVI) and the least-squares iterative algorithm (LSIA) is proposed to extract the phase from two-frame blind phase-shifting interferograms. This method first evaluates the phase shift between two interferograms by the EVI algorithm, and then constructs the fitted interferogram by the addition of two interferograms after filtering the corresponding background intensities, so the phase with high precision can be retrieved by combining two real interferograms and this fitted interferogram using the LSIA method. The proposed algorithm expands the flexibility of the LSIA method and has the high-precision performance compared with the existing algorithms in two-step PSI. Simulation and experiment are performed to verify the feasibility of the proposed algorithm.
\end{abstract}

Keywords: interferometry, phase retrieval, fringe analysis.

\section{Introduction}

Phase-shifting technique has been widely used not only in single-wavelength interferometry [ $\underline{1}-\underline{4}]$ but also in dual-wavelength interferometry [ $\underline{5}-\underline{9}]$. In phase-shifting interferometry (PSI), the accuracy of its phase imaging relies on the accuracy of the phase shifts, which are generated by the piezoelectric transducer (PZT). However, the real phase shifts may be different from the preset values due to the errors caused by the miscalibration of PZT and mechanical vibration. Compared with conventional $N$-step $(N \geq 3)$ PSI [10] that requires at least three interferograms with known phase shifts, two-step PSI relaxes the requirements of the calibration and accuracy of the phase shifter [11], and increases the robustness against environmental factors in practical implementation. On the other hand, compared with single-frame interferometry $[\underline{12}, \underline{13}]$, two-step PSI can resolve the phase-sign ambiguity and offer higher measuring accu- 
racy. In [14], a Fourier transform-based two-step phase demodulation method (Kreis) is proposed, in which the phase can be extracted from two interferograms with unknown phase shifts, but this method may be susceptible to noisy fringe patterns. In [15], based on analyzing the fringe direction, a phase retrieval algorithm is presented by using the regularized optical-flow (OF) method. However, this algorithm for retrieving phase is time-consuming due to the iterative operation using the Gauss-Seidel method. In [16], a Gram-Schmidt (GS) orthonormalization algorithm is proposed for the modulating phase extraction, but this algorithm requires more than one fringe in the interferogram and its accuracy of phase extraction can be affected due to the use of two-step approximate algorithm. In [17], a two-shot fringe pattern phase demodulation algorithm is presented by combining the GS algorithm with the Hilbert-Huang transform, improving the performance of the GS algorithm. In [18], the GS algorithm is applied to either null testing or non-null testing modes. In [19], to achieve the phase with high accuracy, a two-step phase demodulation algorithm is proposed based on the extreme value of interference (EVI). However, this method does not work well when the interferograms exist in the heavy noises. In [20], a phase retrieval algorithm is presented based on phase shift estimation in a local mask with a moderate size, but this algorithm related to fringe analysis is time-consuming and complex, and may fail for noisy fringe patterns.

All the existing algorithms cited above are satisfactory methods for phase retrieval in two-step PSI, but these algorithms [14-20] meet challenges since their accuracy of phase extraction is not high enough compared with the least-squares iterative algorithm (LSIA). However, in two-step PSI, it is impossible to retrieve the phase using the LSIA method since it generally requires a minimum of three frame phase-shifting interferograms [21, 22]. To address this problem and improve the measuring accuracy, a new algorithm combining the EVI algorithm and the LSIA method is proposed to extract the phase from two-frame blind phase-shifting interferograms. In this algorithm, the phase shift between two interferograms is firstly evaluated by the EVI algorithm. Second, using this evaluated phase shift, the fitted interferogram is constructed through the addition of two interferograms after filtering the corresponding background intensities. Finally, the phase with high accuracy can be retrieved by combining two real interferograms and this fitted interferogram based on the LSIA method. Following, we will illustrate the principle of the proposed algorithm, and then give its verification by numerical simulations and experiment.

\section{Principle}

Schematic diagram concerning PSI for the proposed algorithm is shown in Fig. 1, in which $\mathrm{M}$ and $\mathrm{BS}$ are reflector and beam splitter, respectively. The illumination laser is split by $\mathrm{BS}_{1}$ into reference and object beams. The object beam illuminates the object, and the phase shifts of reference wave are produced by a phase shifter (PS). The beams 


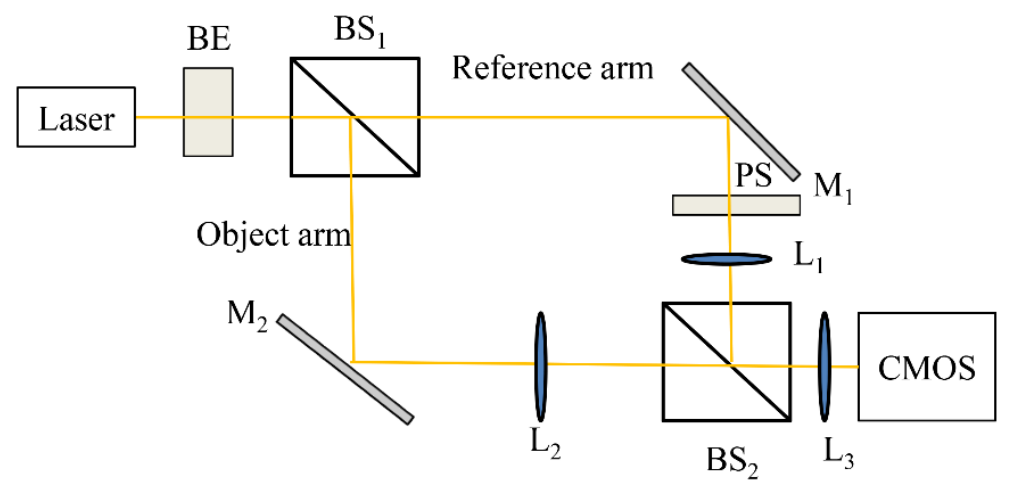

Fig. 1. Schematic diagram concerning PSI for the proposed algorithm. BE: beam expander, $\mathrm{BS}_{1}, \mathrm{BS}_{2}$ : beam splitter, $\mathrm{M}_{1}, \mathrm{M}_{2}$ : mirror, $\mathrm{L}_{1}, \mathrm{~L}_{2}, \mathrm{~L}_{3}$ : lens, and PS: phase shifter.

are combined by $\mathrm{BS}_{2}$, and the resulting interference patterns are recorded by a CMOS camera and finally stored in an image processing system.

In PSI, two frame blind phase-shifting interferograms can be mathematically expressed as

$$
\begin{aligned}
& I_{1 m}=a_{m}+b_{m} \cos \left(\varphi_{m}\right)+\eta_{1 m} \\
& I_{2 m}=a_{m}+b_{m} \cos \left(\varphi_{m}+\delta\right)+\eta_{2 m}
\end{aligned}
$$

where $m=1,2, \ldots, M$, and $M$ is the number of pixels in each frame; $a_{m}, b_{m}$ and $\varphi_{m}$ denote the background intensity, the modulation amplitude and the measured phase of the interferogram in the $m$-th pixel, respectively; $\delta$ (except 0 and $\pi \mathrm{rad}$ ) is the blind phase shift, and $\eta_{1 m}$ and $\eta_{2 m}$ are additive Gaussian noise.

In general, the background intensity $a_{m}$ can be filtered out easily from Eqs. (1) and (2) by using a high-pass Gaussian filter. Thus, Eqs. (1) and (2) become

$$
\begin{aligned}
& I_{1 m}^{\prime}=I_{1 m}-a_{m}=b_{m} \cos \left(\varphi_{m}\right)+\eta_{1 m} \\
& I_{2 m}^{\prime}=I_{2 m}-a_{m}=b_{m} \cos \left(\varphi_{m}+\delta\right)+\eta_{2 m}
\end{aligned}
$$

By combining Eqs. (3) and (4), $\varphi_{m}$ can be retrieved by an arctangent function

$$
\varphi_{m}=\arctan \left(\frac{I_{1 m}^{\prime} \cos \delta-I_{2 m}^{\prime}}{I_{1 m}^{\prime} \sin \delta}\right)
$$

In Eq. (5), as long as the phase shift $\delta$ is known, the measured phase $\varphi_{m}$ can be easily obtained from two interferograms with blind phase shifts. Based on the benchmarking EVI algorithm [19], the phase shift $\delta$ between two interferograms can be evaluated by 


$$
\delta=\frac{1}{S+T}\left[\sum_{i=1}^{S} \arccos \left(\frac{I_{2 m, \mathrm{EVI}_{i}}^{\prime}}{I_{1 m, \mathrm{EVI}_{i}}^{\prime}}\right)+\sum_{j=1}^{T} \arccos \left(\frac{I_{2 m, \mathrm{EVI}_{j}}^{\prime}}{I_{1 m, \mathrm{EVI}_{j}}^{\prime}}\right)\right]
$$

where $I_{1 m, \mathrm{EVI}_{i}}^{\prime}$ is the maximum intensity of $I_{1 m}^{\prime}$ at $i$-th position, $I_{2 m}^{\prime}, \mathrm{EVI}_{i}$ is the intensity of $I_{2 m}^{\prime}$ at the corresponding $i$-th position in $I_{1 m}^{\prime}, \mathrm{EVI}_{i}, I_{1 m, \mathrm{EVI}_{j}}^{\prime}$ is the minimum intensity of $I_{1 m}^{\prime}$ at $j$-th position, $I_{2 m}^{\prime}$, EVI ${ }_{j}$ is the intensity of $I_{2 m}^{\prime}$ at the corresponding $j$-th position in $I_{1 m, \mathrm{EVI}_{j}}^{\prime}$, and $S$ and $T$ are the number of pixels with maximum and minimum intensity in the interferogram $I_{1 m}^{\prime}$, respectively.

In two-step PSI, to further improve the phase retrieval accuracy in Eq. (5) by using the LSIA method, the third interferogram $I_{3 m}$ needs to be constructed firstly. Subsequently, by adding Eqs. (3) and (4), a new equation can be described by

$$
I_{1 m}^{\prime}+I_{2 m}^{\prime}=2 b_{m} \cos (\delta / 2) \cos \left(\varphi_{m}+\delta / 2\right)
$$

Thus, based on Eq. (7), the fitted interferogram $I_{3 m}$ can be constructed as

$$
I_{3 m}=a_{m}+b_{m} \cos \left(\varphi_{m}+\delta / 2\right)=a_{m}+\frac{I_{1 m}^{\prime}+I_{2 m}^{\prime}}{2 \cos (\delta / 2)}
$$

It is noticeable that the phase shift in the fitted interferogram $I_{3 m}$ is about $\delta / 2$. Since there are two real interferograms, $I_{1 m}$ and $I_{2 m}$, and a fitted interferogram $I_{3 m}$, the phase can be extracted by using the LSIA method in two-step PSI. We assume that the background intensity and the modulation amplitude do not vary with frames, then $A_{m}=a_{m}, B_{m}=b_{m} \cos \varphi_{m}$ and $C_{m}=-b_{m} \sin \varphi_{m}$ can be calculated by

$$
\left[\begin{array}{l}
A_{m} \\
B_{m} \\
C_{m}
\end{array}\right]=\left[\begin{array}{lll}
N & \sum_{n=1}^{N} \cos \delta_{n} & \sum_{n=1}^{N} \sin \delta_{n} \\
\sum_{n=1}^{N} \cos \delta_{n} & \sum_{n=1}^{N} \cos ^{2} \delta_{n} & \sum_{n=1}^{N} \cos \delta_{n} \sin \delta_{n} \\
\sum_{n=1}^{N} \sin \delta_{n} & \sum_{n=1}^{N} \cos \delta_{n} \sin \delta_{n} & \sum_{n=1}^{N} \sin ^{2} \delta_{n}
\end{array}\right]^{-1}\left[\begin{array}{l}
\sum_{n=1}^{N} I_{n m} \\
\sum_{n=1}^{N} I_{n m} \cos \delta_{n} \\
\sum_{n=1}^{N} I_{n m} \sin \delta_{n}
\end{array}\right]
$$

where $n=1,2, \ldots, N$, and $N=3$ is the number of phase-shifted interferograms. In Eq. (9), $\delta_{1}=0, \delta_{2}=\delta$ and $\delta_{3}=\delta / 2$.

The measured phase can be retrieved by

$$
\varphi_{m}=\arctan \left(-C_{m} / B_{m}\right)
$$

Once $\varphi_{m}$ is estimated with correct global sign by using Eq. (10), the phase shifts can be determined with correct direction by using the LSIA method. We assume that 
the background intensity and the modulation amplitude do not have pixel-to-pixel variation, then $A_{n}=a_{m}, B_{n}=b_{m} \cos \delta_{n}$ and $C_{n}=-b_{m} \sin \delta_{n}$ can be calculated by

$$
\left[\begin{array}{l}
A_{n} \\
B_{n} \\
C_{n}
\end{array}\right]=\left[\begin{array}{lll}
M & \sum_{m=1}^{M} \cos \varphi_{m} & \sum_{m=1}^{M} \sin \varphi_{m} \\
\sum_{m=1}^{M} \cos \varphi_{m} & \sum_{m=1}^{M} \cos ^{2} \varphi_{m} & \sum_{m=1}^{M} \cos \varphi_{m} \sin \varphi_{m} \\
\sum_{m=1}^{M} \sin \varphi_{m} & \sum_{m=1}^{M} \cos \varphi_{m} \sin \varphi_{m} & \sum_{m=1}^{M} \sin ^{2} \varphi_{m}
\end{array}\right]^{-1}\left[\begin{array}{l}
\sum_{m=1}^{M} I_{n m} \\
\sum_{m=1}^{M} I_{n m} \cos \varphi_{m} \\
\sum_{m=1}^{M} I_{n m} \sin \varphi_{m}
\end{array}\right]
$$

The phase shifts can be determined by

$$
\delta_{n}=\arctan \left(-C_{n} / B_{n}\right)
$$

The phases and the phase shifts with high accuracy can be simultaneously determined in the proposed algorithm by using Eqs. (10) and (12) as the relative phase shifts reach the convergence limit, which can be expressed as

$$
\max \left|\left(\delta_{n}^{t}-\delta_{1}^{t}\right)-\left(\delta_{n}^{t-1}-\delta_{1}^{t-1}\right)\right|<\varepsilon
$$

where $t$ represents the number of the iterations, and $\varepsilon$ is a preset accuracy requirement.

The whole process of the proposed algorithm can be summarized as follows:

Step 1: Capture two frame blind phase-shifting interferograms.

Step 2: Evaluate blind phase shift $\delta$ using Eq. (6).

Step 3: Construct the fitted interferogram $I_{3 m}$ according to Eq. (8).

Step 4: Calculate the measured phase $\varphi_{m}$ using Eq. (10).

Step 5: Estimate the phase shifts $\delta_{m}$ according to Eq. (12).

Step 6: If the convergence limit in Eq. (13) is satisfied, the proposed algorithm is terminated. Otherwise, repeat Step 4 and Step 5.

\section{Simulations and discussion}

\subsection{The spherical wave-front}

Numerical simulations are performed to test the performance of the proposed algorithm. Two simulated interferograms are generated according to Eqs. (1) and (2) by setting the parameters as follows: the background intensity is $a_{m}=100 \exp \left[-0.04\left(x^{2}+y^{2}\right)\right]$, and the modulation amplitude is $b_{m}=90 \exp \left[-0.04\left(x^{2}+y^{2}\right)\right]$, respectively. In addition, the preset threshold $\varepsilon$ is set as $10^{-4}$.

To verify the effectiveness of the proposed algorithm in retrieving the phase of the spherical wave-front, the measured phase of the spherical wave-front is set as $\varphi_{m}(x, y)$ $=\omega \bullet \pi\left(x^{2}+y^{2}\right)$, where $-1.5 \leq x, y \leq 1.5 \mathrm{~mm}$ and $\omega$ is the fringe number in interferogram. 
a

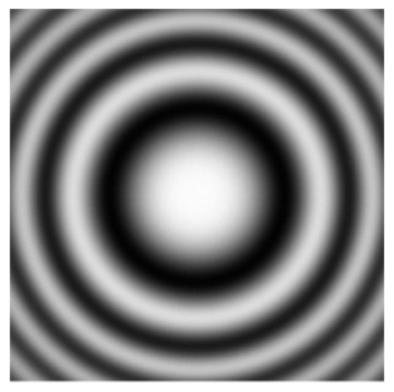

b

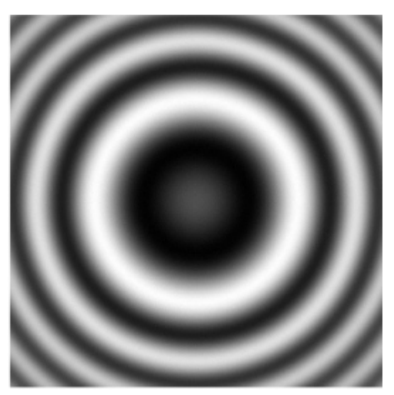

C

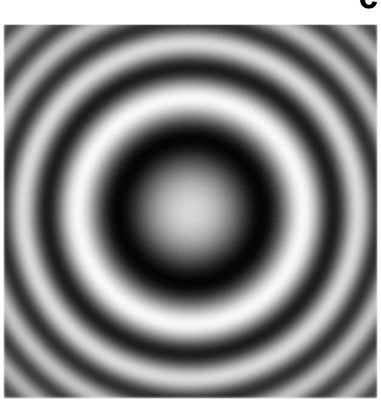

Fig. 2. The spherical wave-front $(\omega=2)$. (a, b) Two simulated phase-shifting interferograms; (c) the fitted interferogram.

Figures $2 \mathbf{a}$ and $2 \mathbf{b}$ respectively present two simulated interferograms of the spherical wave-front $(\omega=2)$ with the size of $300 \times 300$ pixels by setting the parameters as follows: the phase shift $\delta$ is set to $2.0 \mathrm{rad}$; the background intensity is $a_{m}=10 \exp \left[-0.25\left(x^{2}+y^{2}\right)\right]$ +100 , and the modulation amplitude is $b_{m}=100 \exp \left[-0.25\left(x^{2}+y^{2}\right)\right]$, respectively; Gaussian noise with zero-mean and standard deviation $\sigma=1$ is added to the interferograms. After the blind phase shift is calculated by using the EVI algorithm, the fitted interferogram of the spherical wave-front $(\omega=2)$ is constructed based on Eq. (8), as shown in Fig. 2c, and the phase shift in the fitted interferogram is about 1.0028 rad. Subsequently, two real interferograms and the fitted interferogram are addressed with the LSIA method, and then the wrapped and unwrapped phase maps are respectively presented in Figs. 3a using the proposed algorithm. Figures $3 \mathbf{b}$ show the theoretical wrapped and unwrapped phases of the spherical wave-front $(\omega=2)$, respectively.

In order to compare the accuracy of the phase retrieval with different algorithms, the wrapped and unwrapped phases achieved with the EVI, the GS and the LSIA algorithms are respectively illustrated in Figs. 3c-3e. Note that three-frame interferograms with $0,1.5$, and 2.5 phase shifts are employed to extract the phase in the LSIA method.
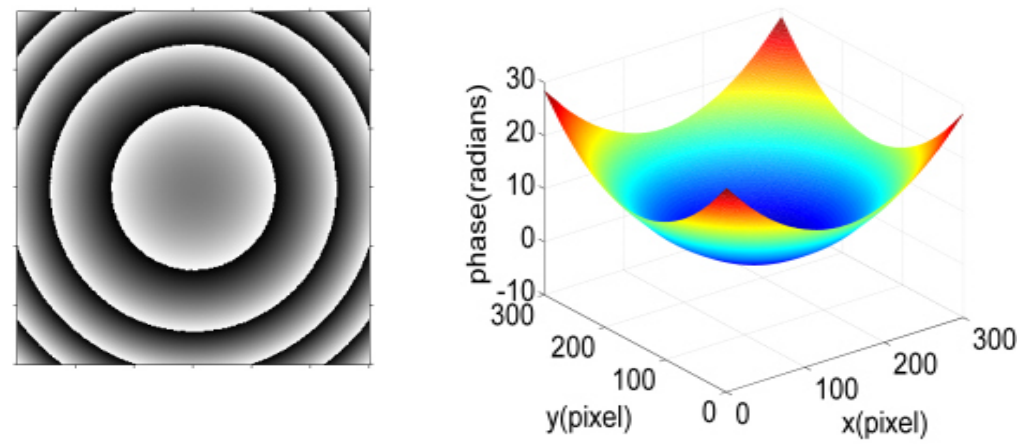

a

Fig. 3. Reconstructed wrapped and unwrapped phases with different algorithms for the spherical wave -front $(\omega=2)$. (a) The proposed algorithm, (b) theoretical calculation, (c) the EVI algorithm, (d) the GS algorithm, and (e) the LSIA algorithm. 

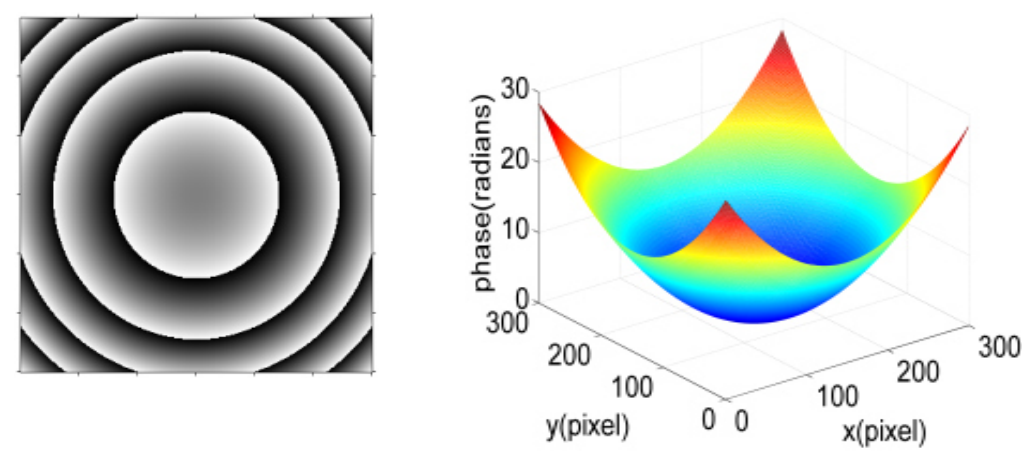

b
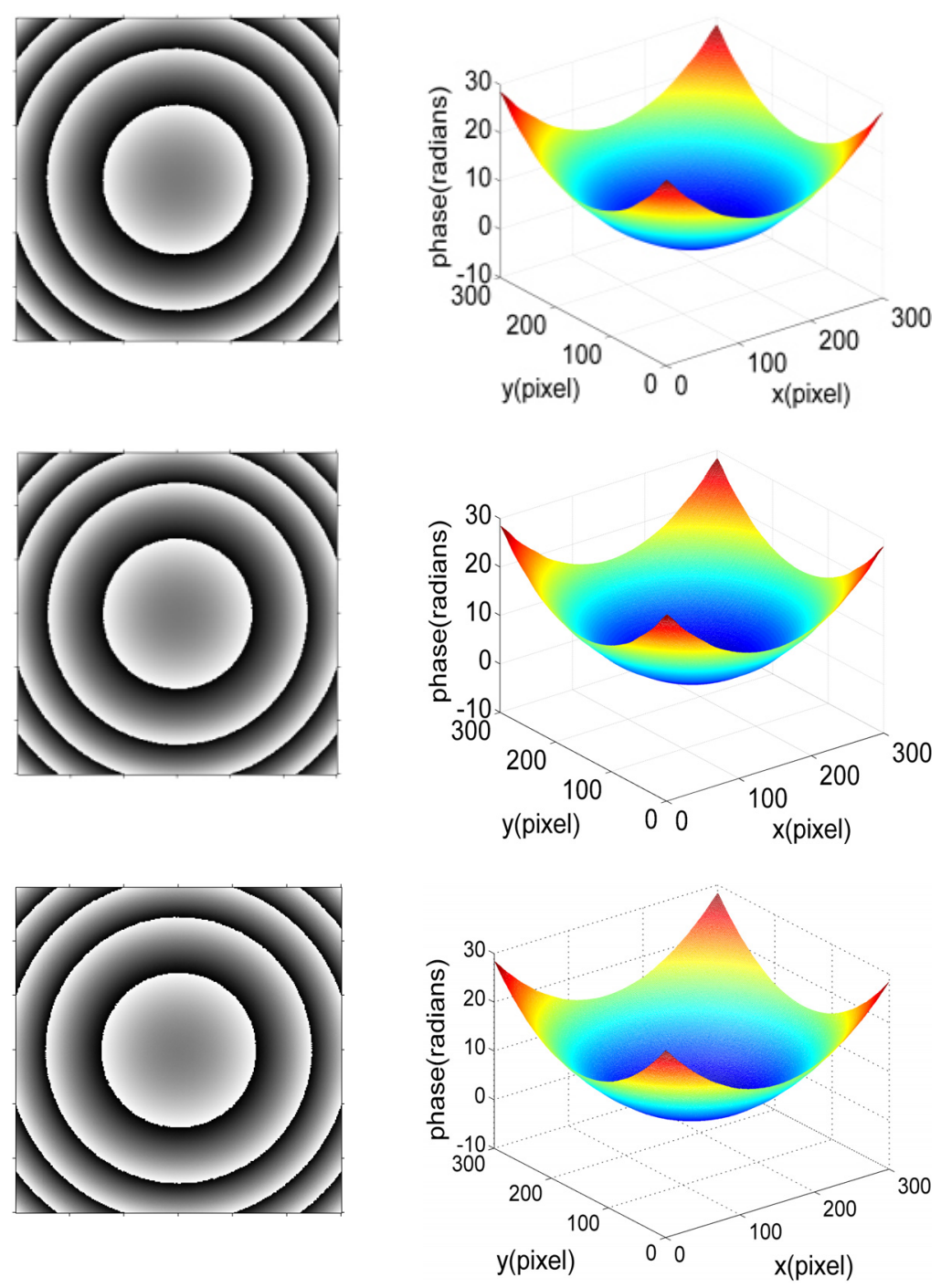

Fig. 3. Continued. 
The simulation results show that the root mean square errors (RMSEs) of the difference between the theoretical phase and the extracted phase with the proposed algorithm, the EVI algorithm, the GS algorithm and the LSIA algorithm are 0.09, 0.13, 0.15 and $0.04 \mathrm{rad}$, and the corresponding processing times in our computer are 5.4, 0.24, 0.2 and $8.9 \mathrm{~s}$, respectively. Due to the use of the LSIA method, the proposed algorithm takes much longer time to retrieve the quantitative phase compared with the EVI and the GS algorithms. But, the accuracy of phase extraction with the proposed algorithm is higher than those with the EVI or GS algorithms since high-frequency noise can be effectively suppressed during the iterations. In addition, the accuracy of phase extraction with the proposed algorithm is lower than that with the LSIA algorithm. The reason is that the LSIA algorithm employs three-frame randomly phase-shifted interferograms to extract the phase while the proposed method only uses two phase-shifted interferograms to retrieve the phase.

To test the robustness of the proposed algorithm, we also investigate the influences of the fringe numbers in interferograms, standard deviations of zero-mean Gaussian noise,

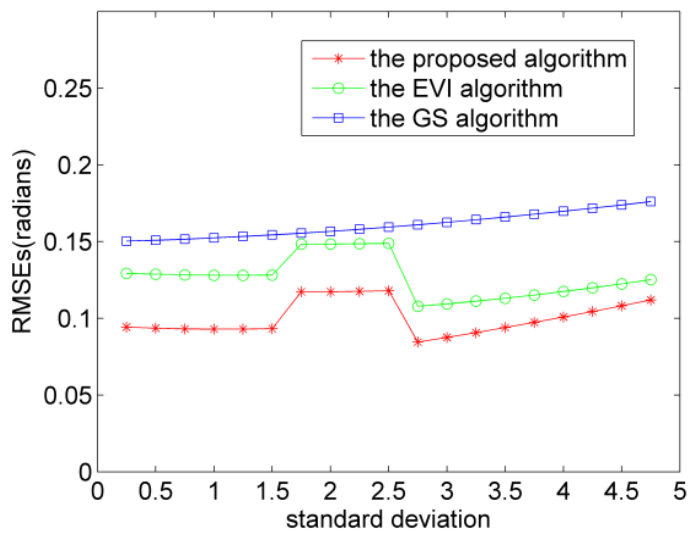

a

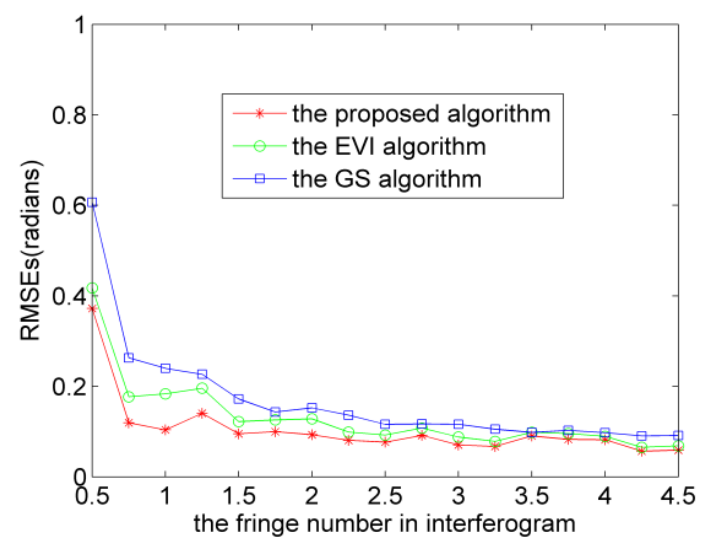

b

Fig. 4. RMSEs of phase extraction for the spherical wave-front with these three algorithms. (a) Corresponding to different standard deviations of zero-mean Gaussian noise, (b) corresponding to different fringe numbers in interferograms, and (c) corresponding to different phase shifts. 


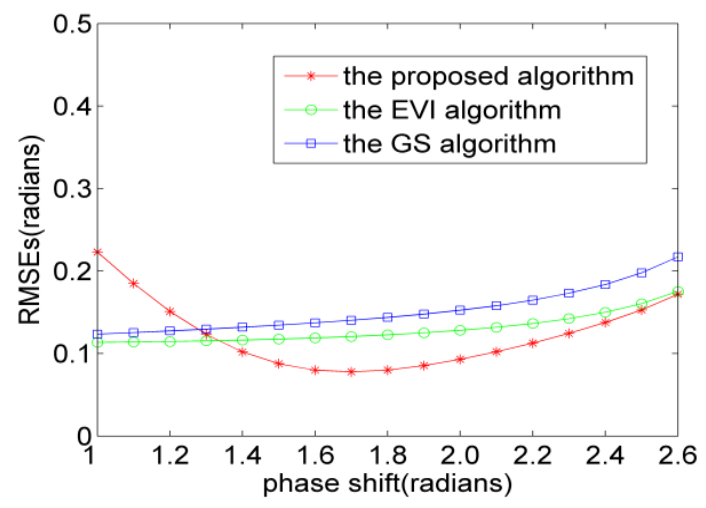

C

Fig. 4. Continued.

and phase shift values of the RMSEs of phase retrieval, as shown in Figs. 4a-4c, respectively. Figures $4 \mathbf{a}$ and $4 \mathbf{b}$ respectively show that when $\sigma$ increases from 0.25 to 4.75 or $\omega$ varies from 0.5 to 4.5 , the RMSEs of phase retrieval with the proposed algorithm are always smaller than those with the EVI or GS algorithms. In Fig. 4c, compared with the EVI and GS algorithms, we can see that when the phase shift value is changed from 1 to $1.3 \mathrm{rad}$, the proposed algorithm has the higher RMSEs of phase retrieval; while the phase shift value is changed from 1.3 to $2.6 \mathrm{rad}$, the proposed algorithm has the smaller RMSEs of phase retrieval. Moreover, it can be seen from Fig. $4 \mathbf{c}$ that the ideal phase shift value is close to $1.7 \mathrm{rad}$ in our proposed algorithm. As a result, we can know that the proposed algorithm is robust and has the high-precision performance.

\subsection{The complex wave-front}

To demonstrate the feasibility of the proposed algorithm in extracting the phase of the complex closed-fringe patterns, Figs. $5 \mathbf{a}$ and $5 \mathbf{b}$ present two simulated fringe patterns of the complex wave-front by setting the parameters as follows: the phase shift $\delta$ is set to $2.4 \mathrm{rad}$; the background intensity is $a_{m}=20 \exp \left[-0.25\left(x^{2}+y^{2}\right)\right]+100$, and the mod-

a

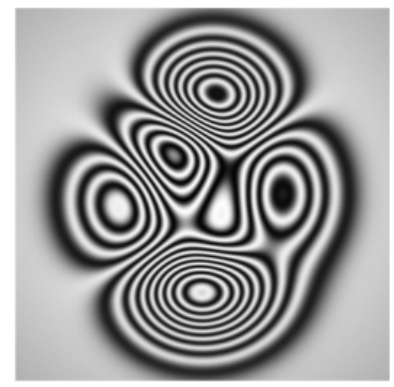

b

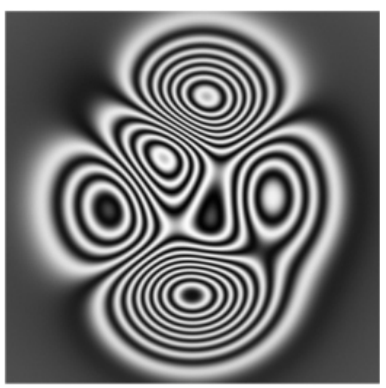

C

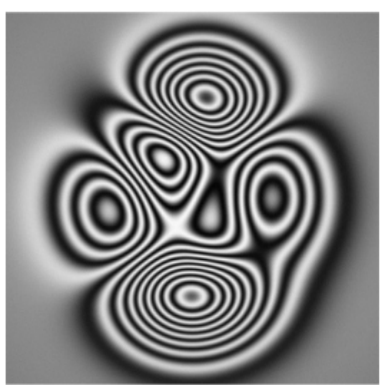

Fig. 5. The complex wave-front. (a, b) Two simulated phase-shifting interferograms, and (c) the fitted interferogram. 
ulation amplitude is $b_{m}=120 \exp \left[-0.25\left(x^{2}+y^{2}\right)\right]$, respectively; Gaussian noise with zero-mean and standard deviation $\sigma=1$ is added to the interferograms. After filtering out the background intensities in Eqs. (1) and (2), the blind phase shift is firstly evaluated through the EVI algorithm, and then the fitted interferogram of the complex wave-front is shown in Fig. 5c. The theoretical wrapped and continuous phases of the complex wave -front are shown in Figs. 6a. We also illustrate the corresponding phases in Figs. $6 \mathbf{b}-6 \mathbf{d}$ with different algorithms, respectively. Through the calculation, the RMSEs of phase
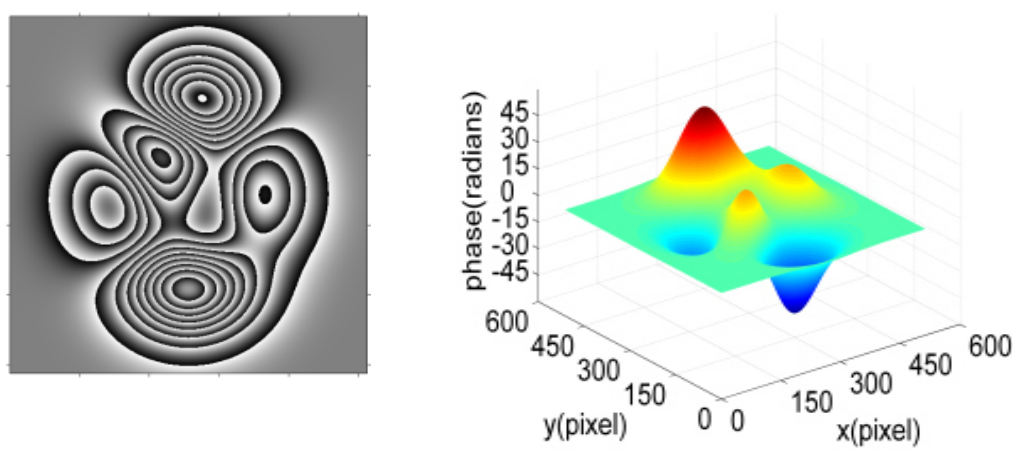

a
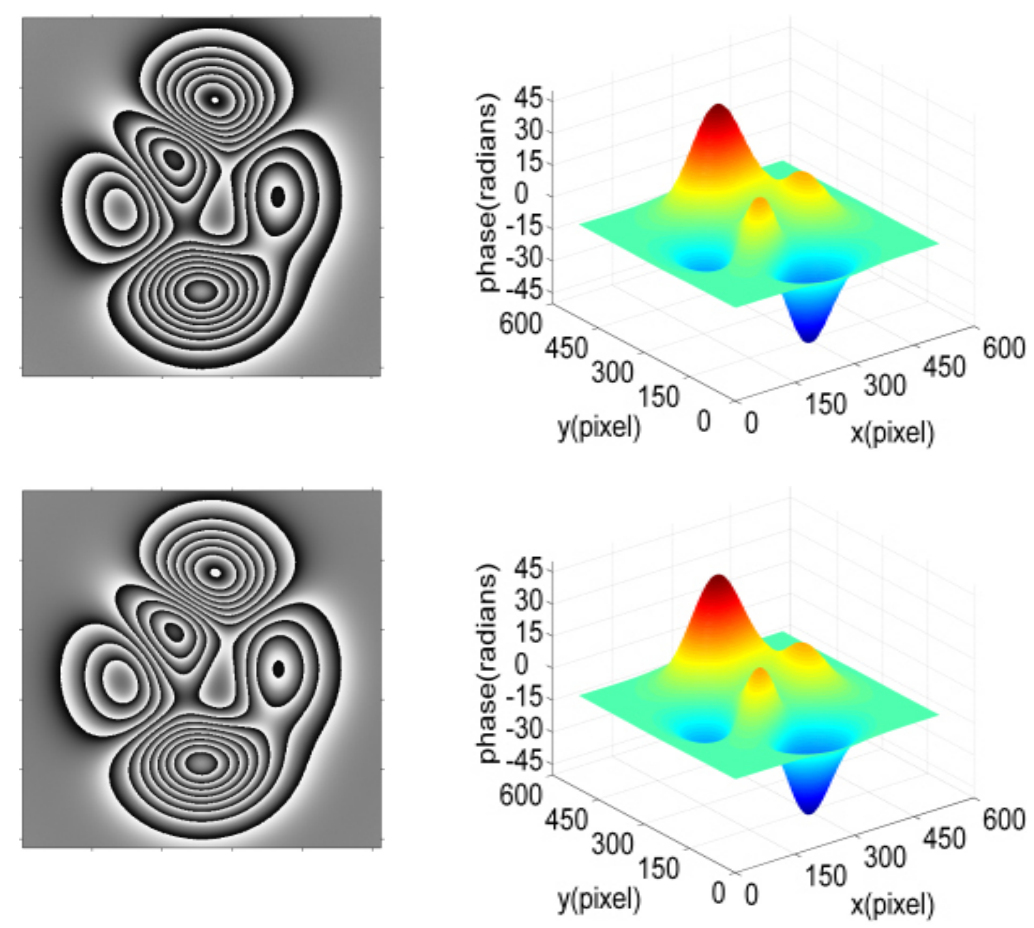

Fig. 6. Reconstructed wrapped and continuous phases with different algorithms for the complex wave -front. (a) Theoretical calculation, (b) the EVI algorithm, (c) the proposed algorithm, and (d) the GS algorithm. 

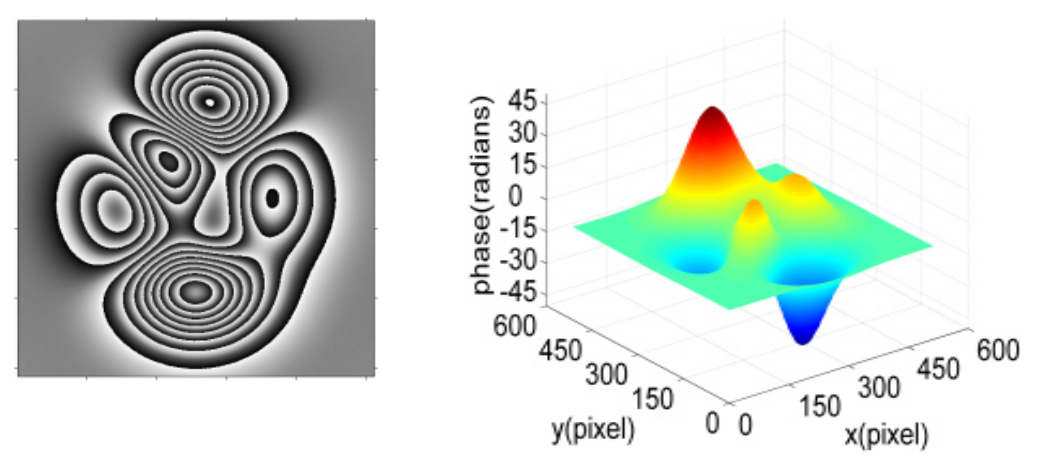

Fig. 6. Continued.

extraction with the proposed algorithm, the EVI algorithm and the GS algorithm are 0.17, 0.23 and $0.26 \mathrm{rad}$, respectively.

In addition, the influences of the background intensity and modulation amplitude on the RMSEs of phase retrieval are also studied, as shown in Figs. 7a and 7b. The background intensity and modulation amplitude are $a_{m}=A_{1} \exp \left[-A_{2}^{2}\left(x^{2}+y^{2}\right)\right]+100$ and $b_{m}=B_{1} \exp \left[-B_{2}^{2}\left(x^{2}+y^{2}\right)\right]$, in which $A_{1}$ and $B_{1}$ are amplitudes and $A_{2}$ and $B_{2}$ are standard deviations, respectively. From Figs. $7 \mathbf{a}$ and $7 \mathbf{b}$, we can find that the RMSEs of phase retrieval almost linearly increase with the increasing of the background intensity amplitude $A_{1}$ from 2 to 20 , except $A_{2}=0.1$. However, the RMSEs of phase retrieval are hardly affected by the modulation amplitude $B_{1}$ inside the range $(102,120)$ since phase calculation is not related to the modulation amplitude term.

Based on the simulations of the spherical and complex wave-fronts above, the advantages of the proposed algorithm can be summarized as: (1) it has a higher precision than the EVI and the GS algorithms; (2) the ideal phase shift is close to $1.7 \mathrm{rad}$; (3) its RMSEs of phase retrieval increase with the increasing of the background intensity am-
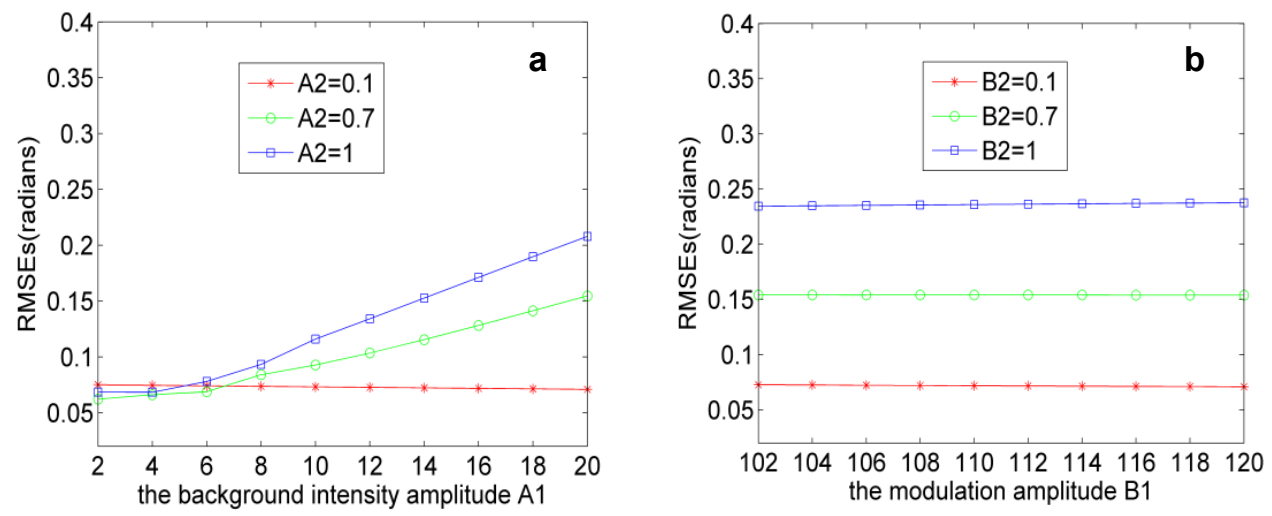

Fig. 7. Influences of different background intensity amplitudes (a), and modulation amplitudes (b) on the RMSEs of phase extraction for the complex wave-front with the proposed algorithm. 
plitude while hardly affected by the modulation amplitude; (4) it is a simple, efficient and robust algorithm.

\section{Experiment}

To demonstrate the practical performance of the proposed algorithm, we performed the optical experiments of the spherical wave-front. The experimental setup is similar to that shown in Fig. 1. A He-Ne laser with the wavelength $\lambda=632.8 \mathrm{~nm}$ is employed as an illumination light source. Note that a quarter-wave plate or a glass plate placed in the reference arm is used as a phase shifter. There is a relationship between the phase shift $\delta$ and the tilt angle $\theta$ of the glass plate, which can be defined as $\delta \approx \pi t(n-1) \theta^{2} /(n \lambda)$. Among which, $t=0.28 \mathrm{~mm}$ and $n=1.55$ are the thickness and refractive index of the glass plate, respectively. Based on this relationship, as the glass plate is rotated by $4^{\circ}$ from its normal direction, the introduced phase shift is about $2.4 \mathrm{rad}$. For the spherical wave-front with the size of $300 \times 300$ pixels, an interferogram without the phase shift as the 1st interferogram is recorded, and two interferograms with the phase shifts introduced by the quarter-wave plate and the glass plate as the 2th and 3th interferograms, are respectively recorded, one of which is presented in Fig. 8a. Following, three phase -shifted interferograms are captured by a CMOS, respectively.
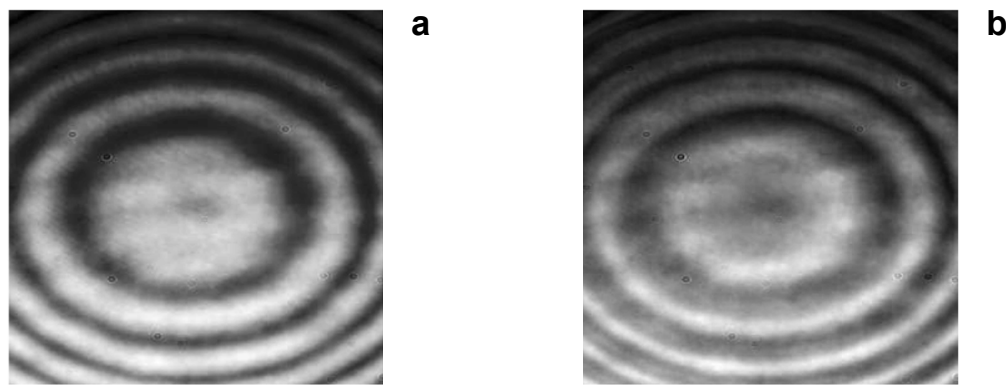

Fig. 8. The spherical wave-front. (a) One-frame real interferogram; (b) the fitted interferogram.
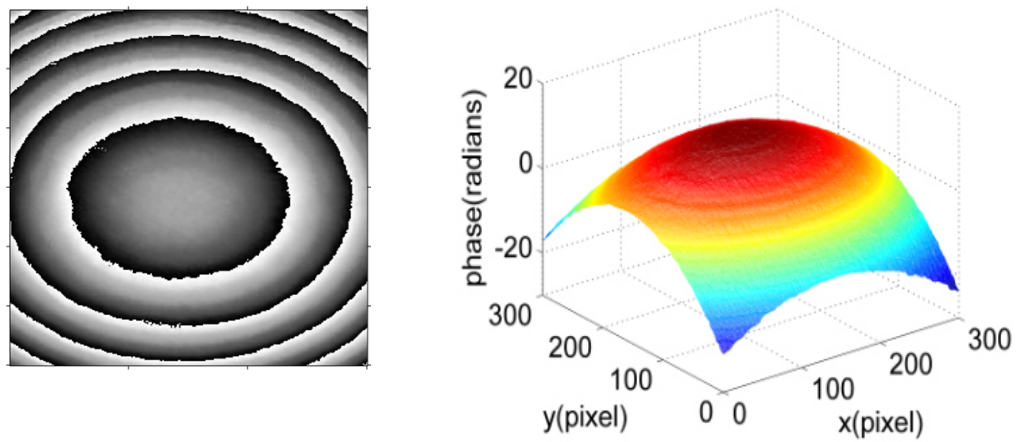

Fig. 9. The spherical wave-front. (a) Reference wrapped and unwrapped phases; reconstructed wrapped and unwrapped phases with (b) the proposed algorithm, (c) the EVI algorithm, and (d) the GS algorithm. 

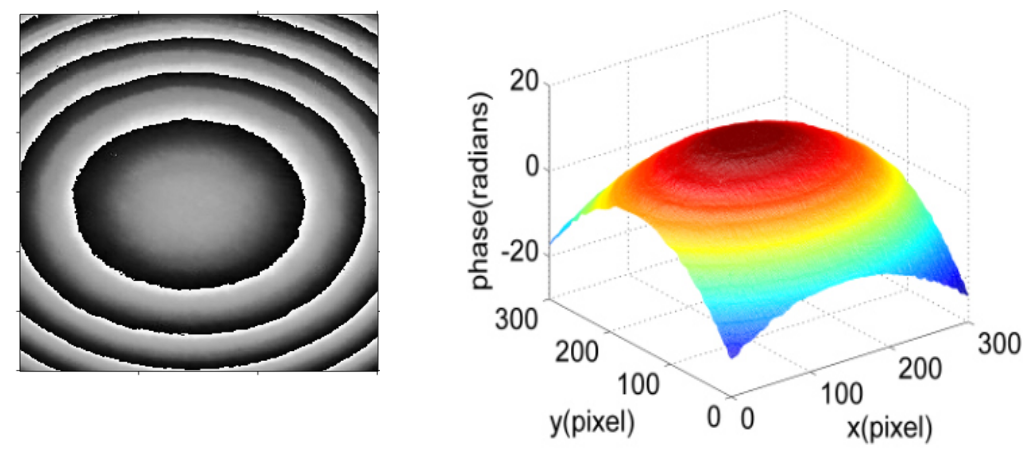

b
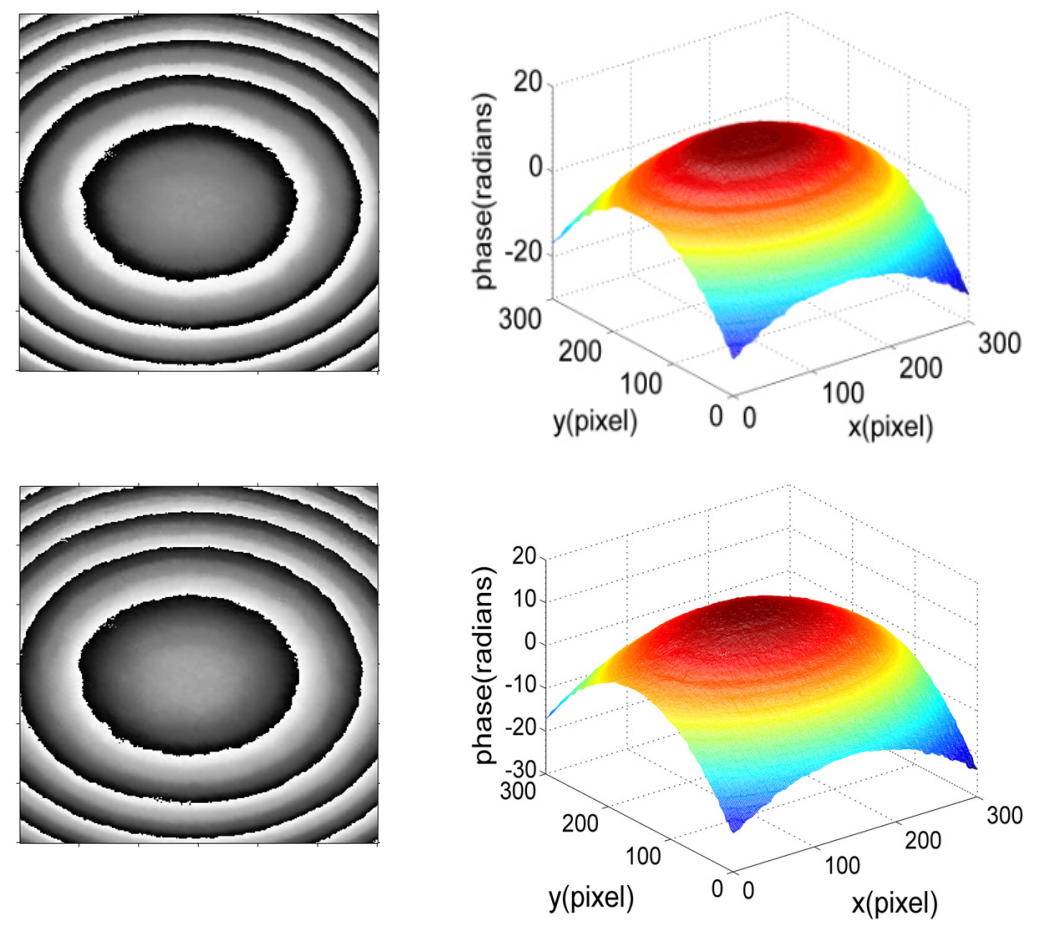

Fig. 9. Continued.

In Figs. 9a, we show the reference wrapped and unwrapped phases of the spherical wave-front obtained by the LSIA method from these three real interferograms. In order to address two blind phase-shifting interferograms in two-step PSI, only two real interferograms are employed to retrieve the phases in the proposed, EVI and GS algorithms. Based on the extracted phase shift using Eq. (6), the fitted interferogram for the spherical wave-front is constructed, as shown in Fig. $8 \mathbf{b}$. The wrapped and unwrapped phases with the proposed, EVI and GS algorithms are presented in Figs. 9b-9d, respectively. Through the calculation, the RMSEs of phase retrieval with the proposed algorithm, the EVI algorithm and the GS algorithm are $0.16,0.23$ and 0.24 rad, respectively. From 
experimental results of the spherical wave-fronts, it can be seen that the proposed algorithm has the smaller RMSE of phase retrieval, compared with the EVI and GS algorithms.

\section{Conclusions}

In summary, to extract the phase from two-frame blind phase-shifting interferograms, a new algorithm combining the EVI algorithm and the LSIA method has been proposed in this paper. In this algorithm, the phase shift between two interferograms is first estimated with the EVI algorithm. Second, using this estimated phase shift, the fitted interferogram is constructed by adding two interferograms after filtering the corresponding background intensities. Finally, based on the LSIA method, the high-precision phase can be extracted by combining two real interferograms and this fitted interferogram. The proposed algorithm not only inherits the advantages of the EVI algorithm, but also expands the flexibility of the LSIA method. Simulation and experimental results show that the proposed algorithm has the high accuracy of phase retrieval compared with the existing algorithms, such as the EVI and the GS algorithms. In a word, our proposed algorithm offers a useful tool for high-precision phase retrieval in two-step PSI.

Acknowledgements - This work was supported by Changzhou Sci\&Tech Program (Grant No. CJ20210032), by the cultivation object of major scientific research project in Changzhou Vocational Institute of Mechatronic Technology (No. 2020ZDXM003), and by National Natural Science Foundation of China (No. 11874184).

\section{References}

[1] Yamaguchi I., Zhang T., Phase-shifting digital holography, Optics Letters 22(16), 1997, pp. 1268 -1270, DOI: $10.1364 /$ OL.22.001268.

[2] Mosino J.F., Servin M., Estrada J.C., Quiroga J.A., Phasorial analysis of detuning error in temporal phase shifting algorithms, Optics Express 17(7), 2009, pp. 5618-5623, DOI: 10.1364/OE.17.005618.

[3] De Groot P., Deck L., Surface profiling by analysis of white-light interferograms in the spatial frequency domain, Journal of Modern Optics 42(2), 1995, pp. 389-401, DOI: 10.1080/09500349514550341.

[4] Meng X.F., Cai L.Z., Xu X.F., Yang X.L., Shen X.X., Dong G.Y., Wang Y.R., Two-step phase-shifting interferometry and its application in image encryption, Optics Letters 31(10), 2006, pp. 1414-1416, DOI: $10.1364 /$ OL.31.001414.

[5] XU X., WANG Y., XU Y., JIN W., Dual-wavelength in-line phase-shifting interferometry based on two dc-term-suppressed intensities with a special phase shift for quantitative phase extraction, Optics Letters 41(11), 2016, pp. 2430-2433, DOI: 10.1364/OL.41.002430.

[6] Kühn J., Colomb T., Montfort F., Charrière F., Emery Y., Cuche E., Marquet P., Depeursinge C., Real-time dual-wavelngth digital holographic microscopy with a single hologram acquisition, Optics Express 15(12), 2007, pp. 7231-7242, DOI: 10.1364/OE.15.007231.

[7] Xu X., WANG Y., JI Y., XU Y., XIE M., HaN H., A novel dual-wavelength iterative method for generalized dual-wavelength phase-shifting interferometry with second-order harmonics, Optics and Lasers in Engineering 106, 2018, pp. 39-46, DOI: 10.1016/j.optlaseng.2018.02.007.

[8] AbDelsalam D.G., KIm D., Two-wavelength in-line phase-shifting interferometry based on polarizing separation for accurate surface profiling, Applied Optics 50(33), 2011, pp. 6153-6161, DOI: 10.1364/ A0.50.006153. 
[9] XU X., WANG Y., XU Y., JIN W., Simultaneous measurement of refractive index and thickness for optically transparent object with a dual-wavelength quantitative technique, Optica Applicata 46(4), 2016, pp. 597-605, DOI: 10.5277/oa160407.

[10] Creath K., V Phase-Measurement Interferometry Techniques, Progress in Optics 26, 1998, pp. 349 -393, DOI: $10.1016 /$ S0079-6638(08)70178-1.

[11] Hariharan P., Phase-shifting interferometry: minimization of systematic errors, Optical Engineering 39(4), 2000, pp. 967-969, DOI: 10.1117/1.602443.

[12] Kemao Q., Two-dimensional windowed Fourier transform for fringe pattern analysis: principles, applications and implementations, Optics and Lasers in Engineering 45(2), 2007, pp. 304-317, DOI: 10.1016/j.optlaseng.2005.10.012.

[13] Tian C., Chen X., Liu S., Modal wavefront reconstruction in radial shearing interferometry with general aperture shapes, Optics Express 24(4), 2016, pp. 3572-3583, DOI: 10.1364/OE.24.003572.

[14] Kreis T.M., JuePTNER W.P.O., Fourier transform evaluation of interference patterns: demodulation and sign ambiguity, Proceedings of SPIE 1553, 1992, pp. 263-273, DOI: 10.1117/12.135312.

[15] Vargas J., Quiroga J.A., Sorzano C.O.S., Estrada J.C., Carazo J.M., Two-step interferometry by a regularized optical flow algorithm, Optics Letters 36(17), 2011, pp. 3485-3487, DOI: 10.1364/ OL.36.003485.

[16] Vargas J., Quiroga J.A., Sorzano C.O.S., Estrada J.C., Carazo J.M., Two-step demodulation based on the Gram-Schmidt orthonormalization method, Optics Letters 37(3), 2012, pp. 443-445, DOI: $10.1364 /$ OL.37.000443.

[17] Trusiak M., Patorski K., Two-shot fringe pattern phase-amplitude demodulation using Gram-Schmidt orthonormalization with Hilbert-Huang pre-filtering, Optics Express 23(4), 2015, pp. 4672-4690, DOI: $10.1364 / \mathrm{OE} .23 .004672$.

[18] Tian C., LiU S., Two-frame phase-shifting interferometry for testing optical surfaces, Optics Express 24(16), 2016, pp. 18695-18708, DOI: 10.1364/OE.24.018695.

[19] Deng J., Wang H., Zhang F., Zhang D., Zhong L., Lu X., Two-step phase demodulation algorithm based on the extreme value of interference, Optics Letters 37(22), 2012, pp. 4669-4671, DOI: $\underline{10.1364 / \text { OL.37.004669. }}$.

[20] Tian C., Liu S., Phase retrieval in two-shot phase-shifting interferometry based on phase shift estimation in a local mask, Optics Express 25(18), 2017, pp. 21673-21683, DOI: 10.1364/OE.25.021673.

[21] Wang Z., Han B., Advanced iterative algorithm for phase extraction of randomly phase-shifted interferograms, Optics Letters 29(14), 2004, pp. 1671-1673, DOI: 10.1364/OL.29.001671.

[22] WANG Z., HAN B., Advanced iterative algorithm for randomly phase-shifted interferograms with intra- and inter-frame intensity variations, Optics and Lasers in Engineering 45(2), 2007, pp. 274 -280, DOI: 10.1016/j.optlaseng.2005.11.003. 